\title{
Manifold adaptation for constant false alarm rate ship detection in South African oceans
}

\author{
C. P. Schwegmann, W. Kleynhans and B. P. Salmon
}

\begin{abstract}
The detection of ships at sea is a difficult task made more so by uncooperative ships, especially when using transponder based ship detection systems. Synthetic Aperture Radar imagery provides a means of observation independent of the ships cooperation and over the years a vast amount of research has gone into the detection of ships using this imagery. One of the most common methods used for ship detection in Synthetic Aperture Radar imagery is the Cell-Averaging Constant False Alarm Rate prescreening method. It uses a scalar threshold value to determine how bright a pixel needs to be in order to be classified as a ship and thus inversely how many false alarms are permitted. This paper presents by a method of converting the scalar threshold into a threshold manifold. The manifold is adjusted using a Simulated Annealing algorithm to optimally fit to information provided by the ship distribution map which is generated from transponder data. By carefully selecting the input solution and threshold boundaries, much of the computational inefficiencies usually associated with Simulated Annealing can be avoided. The proposed method was tested on six ASAR images against five other methods and had a reported detection accuracy of $\mathbf{8 5 . 2} \%$ with a corresponding false alarm rate of $1.01 \times 10^{-7}$.
\end{abstract}

Index Terms-Constant False Alarm Rate (CFAR), Marine technology, Simulated annealing, Synthetic aperture radar (SAR)

\section{INTRODUCTION}

$\mathbf{M}$ ARITIME surveillance is an integral part of Maritime Domain Awareness (MDA). The surveillance of ships at sea is important for a variety of concerns including those related to the environment, commerce and security [1], [2]. With over 100000 ships active at any given point in time in the ocean [1], the detection of ships using any means possible is important. Traditionally, ship monitoring relies on using a transponder system [3]-[6]. These transponder systems range from terrestrial based systems such as Automatic Identification System (AIS) to space-based transceiver systems such as Satellite-AIS (Sat-AIS) and Long Range Identification and Tracking (LRIT) [4]-[6]. Ship detection using transponders becomes difficult when either the transponders are sabotaged or out of range (for terrestrial transponder systems). Despite this, the immense amount of data provided by transponders can still be useful for the creation of a ship distribution map to profile ship movement behavior [4].

C. P. Schwegmann and W. Kleynhans are with the Department of Electrical, Electronic and Computer Engineering, University of Pretoria as well as the Remote Sensing Research Unit, Meraka Institute, CSIR, Pretoria, South Africa. Email: cschwegmann@csir.co.za. Telephone: +27 128413207.

B.P Salmon is with the School of Engineering, University of Tasmania, Australia as well as the Remote Sensing Research Unit, Meraka Institute, CSIR, Pretoria, South Africa.
Maritime surveillance can be accomplished by analyzing Synthetic Aperture Radar (SAR) imagery which is independent from the required cooperation of the ships using transponders. SAR imagery can have large swath widths, day or night observation of an area under most weather conditions and the distinct features of ships over the ocean made it an attractive option for ship detection [3]-[8].

Ship (or target) detection in SAR imagery takes the form of a multistage approach whereby each stage provides ever stricter requirements on the ships that are accepted [5][11]. Central to these detection systems is the stage known as the ship prescreening which uses global or local means of highlighting ships. One of the most prevalent prescreening stages is known as the Cell Averaging Constant False Alarm Rate (CA- CFAR) approach [4], [7], [8]. It is based on a scalar threshold value which determines how much brighter a pixel must be to its local surroundings to be selected as a target or ship. Among the benefits of the CA-CFAR prescreening stage is its low complexity and the ability to compute good initial estimates of the target without computing the probability density function (PDF) for each sub-window. The disadvantage of using a single scalar threshold to define the distribution of reflectance of the ocean is when the distribution of the sea clutter is heterogeneous [11]. An empirical PDF can then be computed for each sub-window to estimate if a pixel matches the target criteria or not [7], [11]. In this paper the assumption that a singular scalar threshold value is sufficient is discarded by creating a constrained threshold manifold whereby each pixel is assigned its own specific threshold. This threshold manifold or constrained surface [12]-[14], in addition to the local statistics within each window, provides a ship detection method which extends the CA-CFAR method to be more flexible whilst still avoiding the local PDF computation that other methods require [10], [11].

The task is then to compute appropriate thresholds for each pixel. For areas with higher average backscatter (those close to the nadir position) lower thresholds may be necessary whilst those further away from the nadir might require higher threshold values. To aid in the selection of threshold values across the manifold, a novel usage of Simulated Annealing (SA) [15], in combination with a ship distribution map, is presented here. Despite the efficiency of methods such as Genetic Algorithms (GA) or Particle Swarm Optimization (PSO), the SA produces a solution which is more intuitive to the problem of computing the threshold manifold [16][18]. The reason is that the CA-CFAR produces accurate initial conditions for the SA to adapt quickly to an acceptable solution, which mitigates the computational inefficiency. 


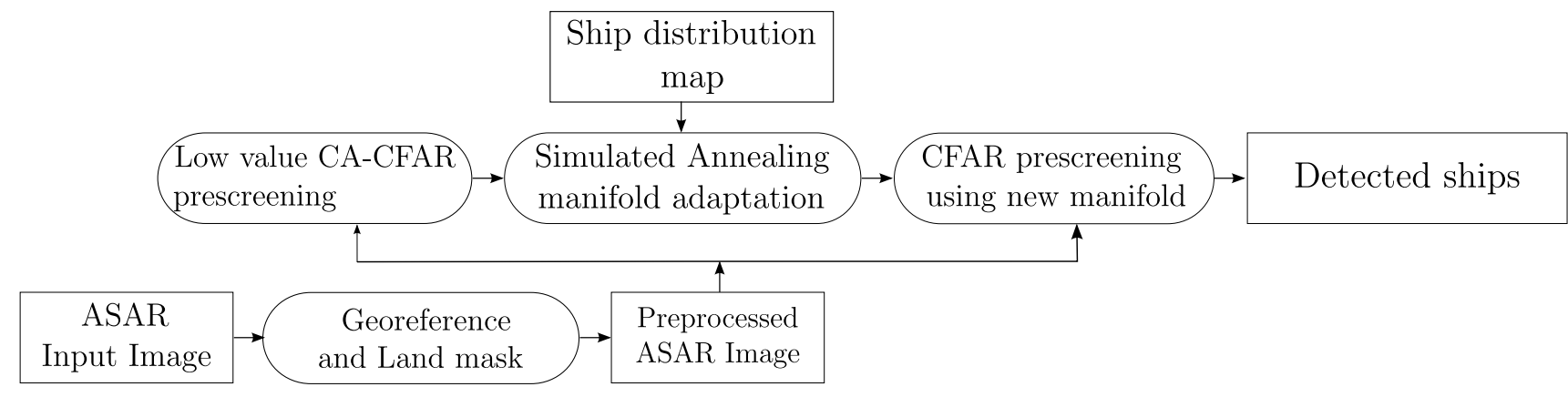

Fig. 1. Flow diagram of the system process for this experiment. An ASAR image is preprocessed by removing the land in the image and georeferencing the image. This processed image is then prescreened using an initial CA-CFAR which is adjusted using SA. The initial solution is adjusted until there is little change in the solution. This is then used in a second prescreening stage to produce a binary image with the detected ships centers as true values.

The organization of the paper is as follows: section II describes the two source of data used. Section III and IV describe the system process and components used to detect ships and section $\mathrm{V}$ presents the results and a discussion thereof. Section VI concludes the paper with closing arguments related to the method described herein.

\section{DATA DESCRIPTION}

\section{A. Synthetic Aperture Radar Imagery}

Six SAR images around the coast of South Africa were used in this study. The SAR images were acquired from ENVISAT's ASAR sensor between February 2012 and April 2012 in VVpolarized Wide Scan Mode (WSM). Despite the fact that a HH-polarized set of images would have been preferred [19], the current set of images were the only ones available for this study. Each of the SAR images has a corresponding expertly visually verified ground truth image indicating the position of the large ships in the image. The images had a spatial resolution of $75 \mathrm{~m} \times 75 \mathrm{~m}$ with a swath width of approximately $400 \mathrm{~km}$ which restricted the minimum size of the ships. This study provides results only for ships visible at this resolution which could include large fishing trawlers, fish factories, cargo or fuel oil ships.

\section{B. Transponder data}

The method proposed in this paper only requires latitude and longitude points of the ships, which can be extracted data from AIS, SAT-AIS or LRIT data. LRIT data was used in this study. LRIT transponders are required to transmit their position every six hours but can be set up to send 1440 positional messages per day per ship, making the acquisition of hundreds of thousands of points over a year possible. The data used in this study includes approximately 450000 ship Latitudes and Longitudes coordinates, recorded over the time period of 2011/03 to 2012/03 off the coast of South Africa. The data was acquired just before the acquisition of the SAR images and it was assumed that the ship distribution map would not deviate significantly from the movement behavior of the ships within the ASAR imagery.

As mentioned previously, ship or target detection requires a multistage approach. It was assumed that no differentiation between ships and other targets are made and that the system can be extended to include additional processing steps using the ship distribution map in order to separate ships and other targets from the detections if required to do so.

The first stage of most ship detection systems is preprocessing. For this study, two forms of image segmentation were performed. The first involves removing land from the image by separating land and sea pixels. This is done in order to prevent incorrect detections over land as well as to reduce land azimuthal ambiguities near the coast. The second form of segmentation involved grouping nearby pixels into groups so that ships of various sizes could be compared equally. Additional preprocessing steps involving filtering were not applied in order to reduce the possibility of altering the sea clutter statistics as well as to prevent the removal of small ships within the ASAR imagery.

The preprocessing step is followed by the prescreening and ship discrimination stages. The prescreening stage is dedicated to improving the detection accuracy whilst the ship discrimination stage attempts to further reduce the false alarms. The method presented in this paper extends the prescreening stage to perform further false alarm removal in lieu of a ship discrimination stage. The ship detection method presented here uses an initial prescreening stage followed by a second prescreening stage with an adaptive threshold manifold to remove false alarms. The detection system proposed in this paper is shown in Fig. 1. The next section gives a more detailed introduction into CFAR and the classical CFAR method known as CA-CFAR.

\section{CA-CFAR Prescreening}

CA-CFAR prescreening involves selecting a threshold so that the number of false alarms detected is kept constant. One of the first and most widely used versions of the CFAR method is known as the Power Ratio (PR) or Cell-Averaging CFAR (CA-CFAR) [4], [7], [8], [10], [11], [20]. The method uses a set of sliding spatial windows to evaluate each pixel region in the SAR image. The windows estimate the various properties of the current region as shown in Fig. 2. The clutter ring estimates the mean surrounding ocean pixel backscatter value and the region of interest is used to estimate the current pixel or group of pixels' mean value. If a region of interest (ROI) is $T$ times brighter than the surrounding pixels then it is assumed to be a bright ROI (i.e. a ship). 


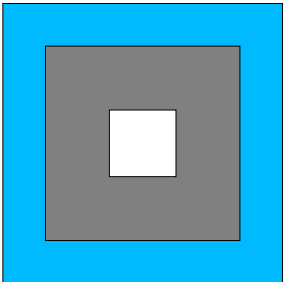

$\square$ Clutter ring $\left(\mu_{\mathrm{C}}\right)$
$\square$ Guard ring
$\square$ Region of Interest $\left(\mu_{\text {ROI }}\right)$

Fig. 2. Pixel neighborhood system used in this study. The mean pixel value inside the clutter and Region of Interest (ROI) rings are calculated as $\mu_{\mathrm{C}}$ and $\mu_{\mathrm{ROI}}$ respectively. The clutter ring is used to represent each pixel's mean ocean backscatter or clutter level. The guard ring is in place to prevent corruption of the clutter mean by objects larger than the ROI. In this study the pixel sizes are: clutter $(7 \times 7)$, guard $(5 \times 5)$ and ROI $(1 \times 1)$.

\section{SAR SHIP DETECTION}

Assuming an input ASAR intensity image $\mathbf{I}$ with image dimensions $X \times Y$ where $x=\{0, \ldots, X-1\}, y=\{0, \ldots, Y-$ $1\}$ and $x, y \in \mathbb{N}$ such that image $\mathbf{I}$ can be defined as [21]

$$
\begin{aligned}
\mathbf{I} & =\left\{\{I(x, y)\}_{x=0}^{x=X-1}\right\}_{y=0}^{y=Y-1} \\
& =\left[\begin{array}{ccc}
I(0,0) & \cdots & I(0, Y-1) \\
I(1,0) & \cdots & I(1, Y-1) \\
\vdots & \ddots & \vdots \\
I(X-1,0) & \cdots & I(X-1, Y-1)
\end{array}\right] .
\end{aligned}
$$

The CA-CFAR prescreening method produces a binary output image $\mathbf{J}(\mathbf{I}, T)$ from the input image defined in (2) using

$$
\mathbf{J}(\mathbf{I}, T)=\left\{\{J(\mathbf{I}, x, y, T)\}_{x=0}^{x=X-1}\right\}_{y=0}^{y=Y-1} .
$$

where $T$ is known as the CA-CFAR threshold and is inversely proportional to the number of false alarms permissible. The CA-CFAR binary image $J(\mathbf{I}, x, y, T)$ is calculated with

$$
J(\mathbf{I}, x, y, T)=\left\{\begin{array}{ll}
\text { true } & \text { if } \mu_{\text {ratio }}(x, y)>T \\
\text { false } & \text { otherwise }
\end{array} .\right.
$$

The quantity $\mu_{\text {ratio }}(x, y)$ is known as the mean (power) ratio and is defined as

$$
\mu_{\text {ratio }}(x, y)=\frac{\mu_{\mathrm{ROI}}(x, y)}{\mu_{\mathrm{C}}(x, y)},
$$

$\mu_{\mathrm{ROI}}(x, y)$ and $\mu_{\mathrm{C}}(x, y)$ are known as the mean region of interest and mean clutter respectively and are calculated using the window system shown in Fig. 2. Notice that the threshold is a single value which acts equally on all pixel values, irrespective of pixel location withing the SAR image. The following section explains how this study extends this single threshold to a threshold manifold to take into account variations in pixel intensity.

\section{A. Extending CA-CFAR Prescreening}

The conventional CA-CFAR method uses a single threshold value to determine if the current pixel ratio $\mu_{\text {ratio }}$ is a ship or not. If the threshold value is low, a vast number of pixels will be highlighted, many of which will be false alarms. If

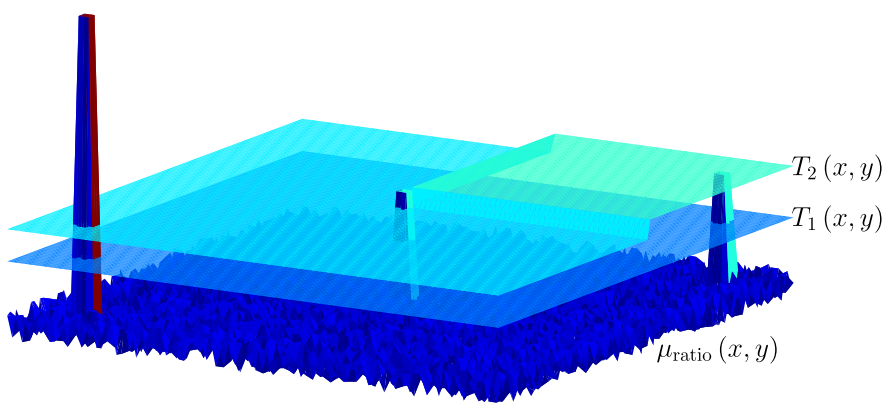

Fig. 3. Ratio image $\mu_{\text {ratio }}(x, y)$ and two threshold manifolds, $T_{1}$ and $T_{2}$. The three spikes indicate three objects that are brighter than their surroundings, with the left two being ships and the right one being a false alarm. Using a flat threshold manifold, the two spikes on the right can not be separated. A non-flat manifold, such as $T_{2}$, allows for discrimination between these two ships.

the threshold value is high then the number of false alarms will be significantly reduced but a number of valid targets will be ignored, causing a drop in detection accuracy. The selection of $T$ in itself is a difficult task made more so by the fact that a single threshold value, even when used to discriminate between local statistics of pixels, may not be sufficient to properly discriminate between similarly valued mean ratio values. Fig. 3 presents an example of how a single threshold (flat manifold) can fail on some occasions to properly discriminate regions with the same or similar $\mu_{\text {ratio }}$ values. Pixels that are brighter than their neighbors manifest as large values compared to others in the neighborhood. These high ratio values appear as "spikes" in the ratio image because $\mu_{\text {ROI }}>\mu_{\mathrm{c}}$. Fig. 3 shows three spikes found in a mean ratio image with two threshold manifolds overlaid - one flat and the other non-flat. A single-valued, flat manifold would not be able to differentiate the two right-hand spikes with the same value. With a flat threshold, either they are both accepted or rejected as bright (ship) pixels. If we assume that the one furtherest right is a false alarm, by extending the threshold manifold to allow for variations in the threshold value along the manifold, the two spikes can easily be separated by increasing the threshold over the one that is not a ship.

The single value $T$ can be extended to a discrete threshold manifold or surface, constrained by the input image dimensions $X \times Y$ where $x=\{0, \ldots, X-1\}, y=\{0, \ldots, Y-1\}$ and $x, y \in \mathbb{N}$ such that threshold manifold $\mathbf{T}$ can be defined as

$$
\begin{aligned}
\mathbf{T} & =\left\{\{T(x, y)\}_{x=0}^{x=X-1}\right\}_{y=0}^{y=Y-1} \\
& =\left[\begin{array}{ccc}
T(0,0) & \cdots & T(0, Y-1) \\
T(1,0) & \cdots & T(1, Y-1) \\
\vdots & \ddots & \vdots \\
T(X-1,0) & \cdots & T(X-1, Y-1)
\end{array}\right]
\end{aligned}
$$

This creates a discrete manifold that is bounded at the sides by the image limits $X$ and $Y$ and threshold value $T \in R^{+}$[12], [13]. In much the same way the various threshold surface solutions possible in this paper all lie within the constrained surface or discrete manifold parametrized by the two positional 
variables $(\mathrm{x}, \mathrm{y})$ and the threshold value $T$ (akin to the two pose variables and azimuthal lighting in [14]).

We introduce a new CA-CFAR prescreening method that differs from previous ones [4], [8], [20]. It can be created by extending (4) to include the new threshold manifold $T(x, y)$. Specifically, the output binary image $J(\mathbf{I}, x, y, T(x, y))$ is calculated with

$$
J(\mathbf{I}, x, y, T(x, y))= \begin{cases}\text { true } & \text { if } \mu_{\text {ratio }}(x, y)>T(x, y), \\ \text { false } & \text { otherwise, }\end{cases}
$$

where $T(x, y) \in R^{+}$. Pixels with an associated threshold value of $T<1.0$ are ignored. The threshold value for this study was assumed to be within the range $T \in[1,255]$. These bounds are important as they significantly reduce the range of possible threshold values. The lower bound of $T \geq 1$ is derived from the fact that ships and thus thresholds need to be at least as bright as their surroundings and the upper bound is derived from the maximum possible pixel value of 255 for an 8-bit input grayscale image. A discussion of how a collection of ship positions can be used unconventionally to help generate threshold manifold values is discussed next.

\section{B. Ship distribution map}

For this study, a total of 450000 ship positions were collected using LRIT transponders between 2011 and 2012 and was used as a priori information to generate a ship distribution map [4]. A small subimage of the ship distribution map off of the South African coast is shown in Fig. 4. Even though LRIT data was used to generate the ship distribution map any source of ship Latitudes and Longitudes can be used to generate the ship distribution map.

If enough ship positions are collected over a number of years, a daily, weekly or even monthly ship distribution map for a given region could be generated. All 12 months of transponder data was used to generate the ship distribution map and it was assumed this would sufficiently model the average movement of ships within the image's geographical limits.

This ship distribution map is used to assign, to each pixel of the input image, a value which represents the likelihood of that pixel having shipping traffic. Pixels with high associated probabilities implies many ships transmitted their position at that position and low associated probabilities means fewer ships had coordinates recorded for that position. More formally, given an input image I with image dimensions defined above, the associated ship distribution map $\mathbf{V}$ for that image is defined as

$$
\begin{aligned}
\mathbf{V} & =\left\{\{V(x, y)\}_{x=0}^{x=X-1}\right\}_{y=0}^{y=Y-1} \\
& =\left[\begin{array}{ccc}
V(0,0) & \cdots & V(0, Y-1) \\
V(1,0) & \cdots & V(1, Y-1) \\
\vdots & \ddots & \vdots \\
V(X-1,0) & \cdots & V(X-1, Y-1)
\end{array}\right] .
\end{aligned}
$$

Each $V(x, y)$ is calculated by adding up the number of ship positions found in the data at that geographical coordinate then

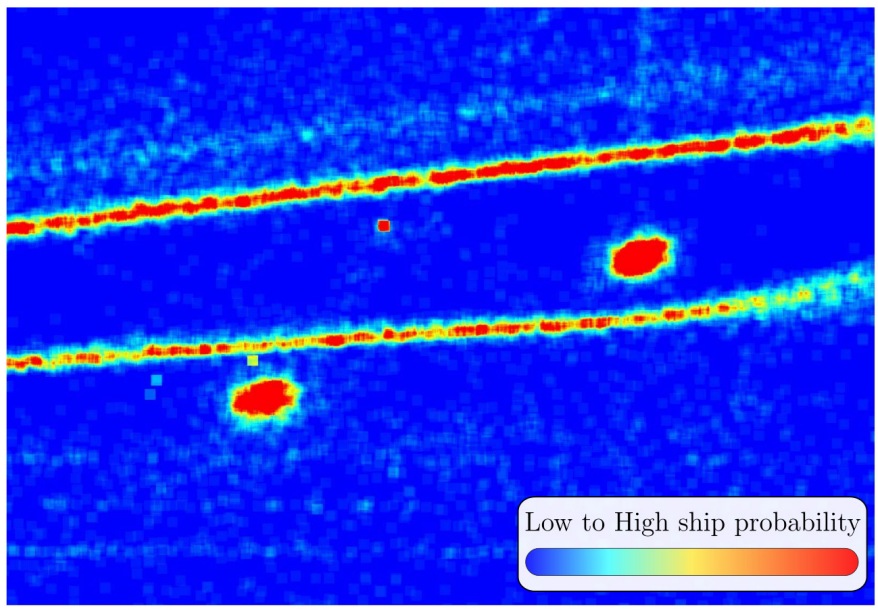

Fig. 4. A section of the ship distribution map generated using all 12 month's LRIT data within the given geographic region. The image shows the ship distribution map off the coast of South Africa, near Mossel Bay (34.1833 $\mathrm{S}, 22.1333^{\circ} \mathrm{E}$ ). It is interesting to note that two shipping lanes are clearly visible as well as two oil rigs. This is due to the fact that thousands of ship positions were collected along those lines, indicating a large number of traversals over those points.

dividing each coordinate by the total number of coordinates counted. This ensures that $\mathbf{V}$ is normalized and that the sum of all $V(x, y)$ is unity. In essence, this forms a 2D histogram of Latitude and Longitude coordinates which are then divided by the total number of coordinates to create a ship distribution map. Once the distribution map is generated, it can be used in a unique way to adapt an initial threshold manifold using Simulated Annealing. If the ship distribution map is unavailable, the current non-flat threshold manifold is passed onto the final stage and is used to threshold the input image to produce a final output. The ship distribution map is therefore useful to significantly reduce the false alarm rate but is not required in order for the method to produce results.

\section{Simulated AnNEALing}

To adapt the threshold manifold a widely used optimization method known as Simulated Annealing was used in conjunction with the ship distribution map [15]. Simulated Annealing mimics the process of heating a material and allowing it to slowly cool to reduce abnormalities in the material. The method works by altering a currently accepted solution, testing the validity of the new solution and then replacing the current best solution with the new solution. The method also allows "bad" solutions to be accepted to improve solution diversity. A flow chart of the Simulated Annealing method is shown in Fig. 5. The benefit of SA is that it uses a given solution to generate further solutions. This is in contrast to other methods, such as GA, which search the entire solution space using multiple different candidates [16], [17]. While other methods focus on searching the entire solution space using different versions of a number of solutions this is not required for SA because if the initial solution is acceptable only further improvements to the input solution are required to get a solution which provides good results. In the case of this study, it is assumed that the initial threshold manifold highlights all ships within the ASAR 


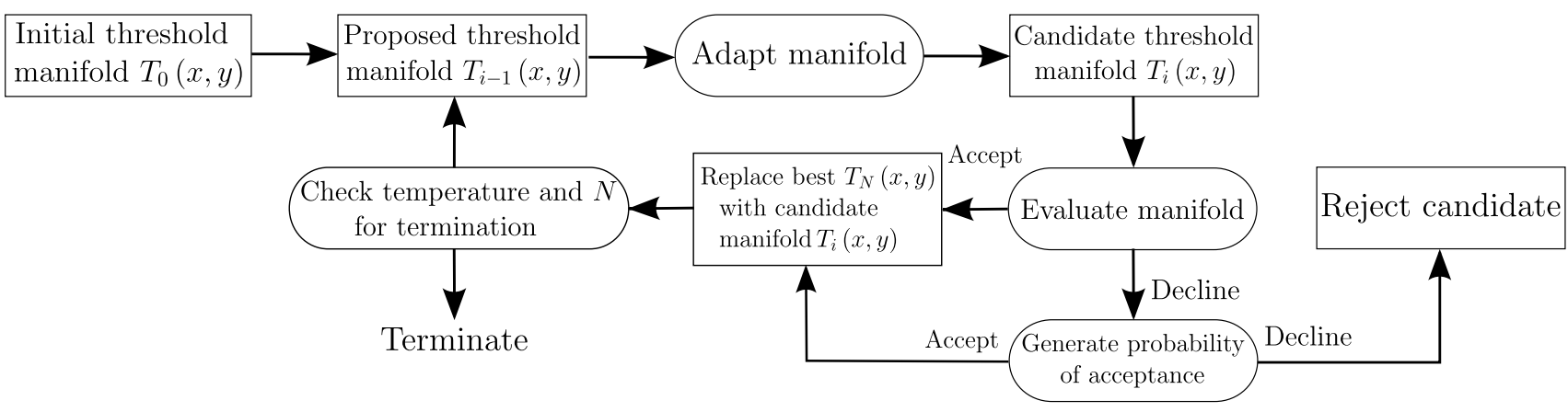

Fig. 5. The Simulated Annealing threshold manifold adaptation process. The process starts at $i=1$ by using the initial manifold $T_{0}$. The manifold is adapted using the process described in section IV-C which generates the candidate threshold manifold. If the candidate threshold manifold fails evaluation it can still be selected as a new, best candidate by means of the Boltzmann probability as described in section IV-C. Finally, the process is terminated when either $N$ steps have occurred or the change in temperature over a number of steps has stayed constant.
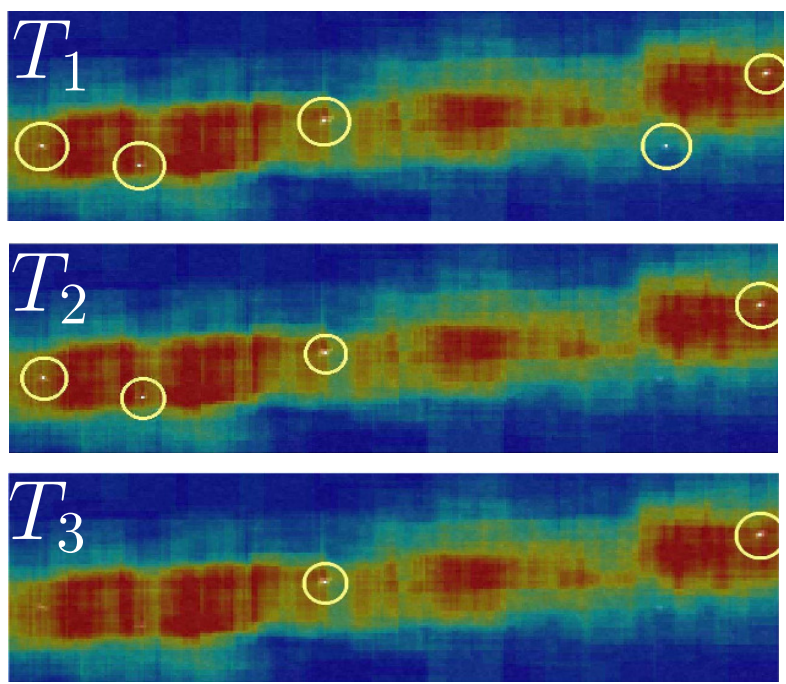

Low to High ship probability CA-CFAR detected ship

Fig. 6. Three flat threshold manifolds are shown with $T_{i}(x, y)=$ $\{2.0,2.5,3.0\}$. The ship distribution map $V(x, y)$ is superimposed over each image. The number of ships are $L_{1}=5, L_{2}=4$ and $L_{3}=2$ and the total probability is $v_{1}^{\text {total }}=0.5, v_{2}^{\text {total }}=0.45$ and $v_{3}^{\text {total }}=0.2$ per step. Using eq. 14 the cost values are $D_{1}=0,900, D_{2}=0,950$ and $D_{3}=0,925$. Notice how, intuitively, the highest cost threshold $T_{2}$ is the best threshold manifold because it removes a redundant bright pixel present at $T_{1}$ but does not remove the two high probability ships like $T_{3}$ does.

imagery. This implies that the initial threshold manifold $T_{0}$ is a good starting solution because subsequent processing steps need only remove the false alarms by increasing those pixels' thresholds to improve performance. This intelligent starting manifold threshold selection significantly reduces the number of searchable ship positions from the entire image to only those as bright as their surroundings. Subsequent steps of the SA processes uses the ship distribution map to evaluate changes in threshold manifold values. For the sake of brevity $T_{i}$ for $i=1,2, \ldots, N$ is equivalent to $T_{i}(x, y)$ where $N$ represents the total number of Simulated Annealing steps.

\section{A. Initial threshold manifold}

The first stage of the Simulated Annealing process is to generate an initial, acceptable solution. The initial threshold manifold, $T_{0}$, is generated by running a low, flat CA-CFAR prescreening on the input ASAR image $\mathbf{I}$ such that $T_{0}(x, y)=$ $J(\mathbf{I}, x, y, T(x, y)=1.0)$. This will select all areas of the input image that have a brighter-than-average pixel value including all ships. These positions will have an associated threshold manifold value of $T_{0}=1.0$ whilst all others will have have $T_{0}=0.0$. To correctly increase threshold manifold values for false alarms a means of threshold manifold evaluation is presented next.

\section{B. Mean change in probability per ship}

Simulated Annealing requires a manner to evaluate the current solution $T_{\text {current }}$ and its possible replacement $T_{i}$. To do so, some metric must be calculated for each solution. Given $T_{i}$ and $\mathbf{I}$, the number of ships detected $L_{i}$, the total probability $v_{i}^{\text {total }}$ at time step $i$ can be calculated. The total probability $v_{i}^{\text {total }}$ is the sum of all probabilities for all the detected ship centers across the whole image using $V(x, y)$ at each time step $i$ which is computed as

$$
v_{i}^{\text {total }}=\sum_{a}^{X} \sum_{b}^{Y}\left\{V(a, b) \mid J_{i}\left(\mathbf{I}, a, b, T_{i}\right)=\text { true }\right\},
$$

where $J_{i}\left(\mathbf{I}, a, b, T_{i}\right)$ is the input image $I(x, y)$ processed using the CA-CFAR with the threshold manifold $T_{i}$. Note that total probability may change at each step because the detected ships may change at each stage.

The mean probability per ship is

$$
\alpha=\frac{v^{\text {total }}}{L_{i}} .
$$

This can be extended to include the variations in the mean probability per ship at each time step by noting the change in $\alpha$ and the change in the number of ships such that the mean change in probability per ship, $\beta_{i}$, is

$$
\beta_{i}=\frac{\left|v_{i}^{\text {total }}-v_{i-1}^{\text {total }}\right|}{\left|L_{i}-L_{i-1}\right|+\epsilon} .
$$


The symbol $\epsilon$ is a arbitrarily small value, typically $\epsilon<<10^{-9}$. At each time step, $\beta_{i}$ can change based on the current threshold manifold $T_{i}$, which then directly affects the value of total probability $v_{i}^{\text {total }}$.

Once the mean change in probability $\beta_{i}$ is calculated for a given time step, a cost function can be used decide if the current threshold manifold $T_{i}$ represents an improvement or not. The cost function at time step $i, D_{i}$, is calculated using $\beta_{i}$ with

$$
D_{i}=1-\left|\beta_{i}-\beta_{i-1}\right| .
$$

For the initial threshold $T_{0}$ values are assumed such that $D_{0}=$ $1, v_{0}^{\text {total }}=0, L_{0}=0$ and $\beta_{0}=0$. Cost function values that are closer to one are preferable because they represent a smaller change in mean ship probability. This is because an small decrease in mean ship probability indicates a reduction in the number of ships in low probability zones which are most likely false alarms. This causes a small change in $\beta$ between step $i-1$ and $i$ and thus the cost function tends closer to one in those cases. An example of this process is shown in Fig. 6. Do note that for the sake of clarity, a flat threshold manifold is used in this example and not the non-flat manifold as introduced in this paper (the same principle applies).

\section{Manifold adaptation}

The manifold adaptation scheme used in this study estimates the degree to which the areas of the manifold should be adapted by using the number of ships that are neighboring every ship. The threshold manifold is increased by a uniform random amount inversely proportional to the number of ships near each ship and added to the previous threshold value at that pixel which is expressed as

$$
\begin{aligned}
T_{i}(a, b)= & \left\{T_{i-1}(a, b)\right. \\
& \left.+\left(R * \frac{1}{Z(a, b)}\right) \mid J_{i}\left(\mathbf{I}, a, b, T_{i}\right)=\text { true }\right\} .
\end{aligned}
$$

$Z(a, b)$ refers to the number of ships in a square area around the ship found in $J_{i}\left(\mathbf{I}, a, b, T_{i}\right)$. If no ships are found within the area, $Z(a, b)=1$. The reasoning behind the inverse relation between the number of ships and the threshold change is that areas with more ships should be increased slowly as the likelihood of ships in those areas is assumed to be more. Singular, solitary pixels' thresholds should be increased rapidly so that their effect on the overall mean probability can be ascertained more quickly. If these solitary pixels are in low probability areas then their removal will have little to no effect on the mean probability per ship and these would typically be assumed as false alarms and require higher threshold values.

Despite the above, a threshold with a low cost can still be accepted if the rejected candidate's probability of acceptance is above a given value known as the Boltzmann probability. The Simulated Annealing method allows for this replacement of the "best" solution in order to prevent the process from settling into non-optimal, local minima. To prevent this, the temperature parameter $\gamma$ is introduced which is related to the mean threshold value. Specifically, the current temperature $\gamma_{i}$ is equal to

$$
\begin{aligned}
\gamma_{i} & =\frac{100}{\mu_{T_{i}}} \\
\mu_{T_{i}} & =\frac{1}{L_{i}} \sum_{x=0}^{x=X-1} \sum_{y=0}^{y=Y-1} T_{i}(x, y) \text { where } T_{i}(x, y)>0,
\end{aligned}
$$

for $i=1 \ldots N$. We assume $\gamma_{0}=100$ and $\mu_{T_{0}}=1.0$ because at $i=0$ all threshold values within $T_{0}$ are either $T=1.0$ for highlighted pixels or $T=0.0$ for the rest.

The value of $\mu_{T_{i}}$ increases as the simulated annealing process continues, thus decreasing the temperature $\gamma_{i}$ over time. Using the current temperature and the change in cost, a previously rejected threshold manifold can be accepted using the Boltzmann probability if

$$
e^{-\frac{\Delta D}{\gamma_{i}}}>R \text {. }
$$

Where $\Delta D$ is the change in cost between the current solution and the best solution, $\gamma_{i}$ is the current temperature of the solution and $R$ is a random uniform real number in the range $[0,1]$.

The above is repeated until the change in cost function over a number of time steps is negligible or a number of predefined time steps $N$ has been reached. The final output image is $J_{N}=J\left(\mathbf{I}, T_{N}(x, y)\right)$. The ships within this image are grouped together, and their center positions within the image are used to compare against the known ship positions to determine the methods performance. Using the center position ensures groups of nearby detections are fused in order to prevent azimuthal ambiguities near to the ships whilst maintaining the correct number of detections.

\section{Proposed method components discussion}

1) Method components: The various components of the method proposed here have been designed in such a manner that a selection of methods can be used in lieu of the ones presented in this paper. The initial threshold manifold can be generated using a number of different techniques including Greatest-of CFAR (GO-CFAR), Smallest-of (SO-CFAR), Order-Statistic CFAR (OS-CFAR) [8] or even by converting unconventional methods to work for ship detection [22]. The final performance of the method as a whole is dependent on the properties of this input method and so it should be selected with care. It is important to select an initial threshold manifold generation method that provides a high detection accuracy to ensure that subsequent steps of the proposed method effectively remove false alarms whilst maintaining correctly detected ships. The SA process can be replaced with alternatives, like a GA-based method if a carefully considered initial and subsequent solution generation scheme is devised. Finally, the type of CFAR method used to prescreening the image (both before and after the manifold has been generated) is flexible and the details of this is discussed next.

2) CA-CFAR and other CFAR variants: Each of the CFAR variants use the clutter adjacent to the test region in different ways. In the CA-CFAR method all of the background values not in the guard window are considered equally and are 
compared to the center pixel or ROI. By weighting each pixel equally no preference towards brighter or darker background pixels are given. Contrastingly, when using one of the other statistical CFAR methods, the representation of the clutter is formed by a single pixel, such as GO-CFAR using the greatest pixel neighbor and SO-CFAR using the smallest pixel neighbor. This neighborhood representation is then compared to the ROI in the same way for the different CFAR methods using a threshold $T$. Conventionally, the distinction between which type of CFAR used is important. This is because the different CFAR types handle different types of clutter better or worse for a given threshold value (or a set probability of false alarm). As mentioned in [8], SO-/GO-/OS-CFAR based methods tend to perform better when applied to heterogeneous clutter. However, the method proposed here extends the definition of the CA-CFAR method to allow the threshold to vary on a per-pixel basis. By doing so, the extended CA-CFAR acts as a variable statistic CFAR method such that for some pixels the threshold can be set low to simulate a SO-CFAR or high for other pixels to simulate a GO-CFAR. The standard CACFAR suffers from being sensitive to either too low or too high values in the background which skews the background statistics and can provide unsatisfactory performance when using a single threshold value. The variability afforded by the threshold manifold offsets the conventional CA-CFAR's downside because darker or brighter pixel neighborhoods are compensated for by changes across the threshold manifold. It should be noted that whilst the CA-CFAR method has been extended here, a number of CFAR forms could also be extended to make use of the variable threshold manifold. The CA-CFAR method used here could be replaced with the SO-/GO-/OS-CFAR based methods by changing how $\mu_{\text {clutter }}$ is calculated. The final threshold manifolds will look considerably different between the methods because each threshold manifold would compensate the difference between the test ROI and background clutter representation differently through the SA process. It is for this reason that only the CA-CFAR and GO-CFAR are used to compare to the proposed method in order to highlight the flexibility provided by the extended CA-CFAR prescreening method.

\section{EXPerimental Results AND Discussion}

The experiments were conducted on six ASAR images with a total of 135 ships across all images. A separate Simulated Annealing manifold adaptation for each of the six images was performed before applying the threshold manifold with the lowest number of false alarms to the input image. Detection accuracy (DA) is the percentage of correctly detected ships given all the possible ships across the entire image. The false alarm rate (FAR) is the number of falsely detected ship pixels divided by the total number of sea pixels tested. Table I and II show the results of the proposed method compared to a conventional CA-CFAR at three different thresholds, a GOCFAR at two different thresholds, a standard and advanced Otsu's based thresholding method [23], [24] and an advanced automatic CA-CFAR threshold selection method presented in [4]. The parameters for the CFAR based methods had ROI, guard and clutter sizes of $1 \times 1,5 \times 5$ and $7 \times 7$ respectively and were selected based on the ship sizes expected. The standard Otsu's method had no parameters but the advanced Otsu's method required a minimum and maximum number of ships as well as the minimum and maximum size of the ships in each image which were set to $2,135,1$ and 7 respectively. For the SA a maximum number of $N=10000$ steps with the initial CA-CFAR prescreening stage using the same parameters as the CA-CFAR method at $T=1.0$ was tested.

Table I illustrates the difficulties of using a single threshold across a number of images when compared to the proposed method. The CA and GO variants of the CFAR prescreening method have comparable DA performances but fail to attain the same performances of the proposed method. Despite the fact that the GO-CFAR variant has fewer false alarms than the CA-CFAR the proposed method has an order of magnitude better FAR and comparable DA as well as more consistent FAR values. When selecting an initial threshold manifold generation method it is important to realise that at $T=1.0$ the GO-CFAR method fails to highlight every ship within the image. Due to the way in which the final solution is obtained this missing ship will not be identified as the SA process continues. The flat threshold selected for the first three images indicate that the CA-CFAR thresholds would be better selected between $T=2.5$ and $T=3.5$ for the CA-CFAR and between $T=1.0$ and $T=1.5$ for the GO-CFAR to achieve a better balance between high DA and low FAR.

Table II illustrates more advanced methods compared to the proposed method. The flat threshold CA-CFAR method can still be effective if an intelligent selection of flat threshold value is made per image, as is the case when using the automatic selection of thresholds from [4]. The results of this method are shown in the first column of Table II. This method uses the ship distribution map to select a flat threshold for each image separately and provides a higher level of DA and correspondingly lower FAR compared to the basic CA-CFAR results. The standard and advanced Otsu's prescreening methods were selected to compare ship detection methods which work differently to the CFAR-based methods. Both Otsu's methods utilize global thresholding and the advanced method uses morphological image operations to remove false alarms. The standard Otsu's performed poorly, with a comparable mean DA of $87.9 \%$ but a mean FAR of $1.015 \times 10^{-2}$. This indicates that a global-based prescreening method provides unacceptable performance and requires significant adjustments and/or an additional ship discrimination stage in order to be comparable to the proposed method. This is taken note of in [23], and this advanced Otsu's uses a morphological based ship discrimination stage to reduce false alarms. Using the selected parameters, the method was able to reduce the mean FAR of the standard Otsu's thresholding whilst maintaining the same mean DA. Despite this, the method failed to reduce a significant number of the false alarms across all images and maintained a high standard deviation for both DA and FAR.

Looking towards the proposed method we note that it had the lowest mean FAR of all the methods tested. Compared to the next best method, the automatic threshold selection method, a drop of $6 \%$ DA was deemed acceptable for the $40 \%$ 
TABLE I

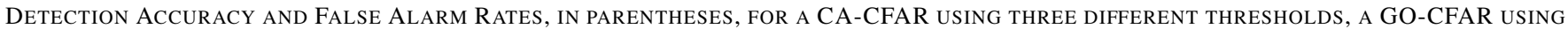
TWO DIFFERENT THRESHOLDS AND THE PROPOSED METHOD.

\begin{tabular}{ccccccc}
\hline Image & CA-CFAR $T=1.0$ & CA-CFAR $T=2.5$ & CA-CFAR $T=3.5$ & GO-CFAR $T=1.0$ & GO-CFAR $T=1.5$ & CA-CFAR SA \\
Image 1 & $100 \%\left(5.170 \times 10^{-3}\right)$ & $82.4 \%\left(1.313 \times 10^{-7}\right)$ & $52.9 \%\left(3.282 \times 10^{-8}\right)$ & $100 \%\left(2.085 \times 10^{-3}\right)$ & $100 \%\left(9.281 \times 10^{-7}\right)$ & $82.4 \%\left(1.050 \times 10^{-8}\right)$ \\
Image 2 & $100 \%\left(5.720 \times 10^{-3}\right)$ & $88.4 \%\left(1.556 \times 10^{-5}\right)$ & $58.1 \%\left(1.186 \times 10^{-5}\right)$ & $100 \%\left(2.516 \times 10^{-3}\right)$ & $100 \%\left(1.911 \times 10^{-6}\right)$ & $83.7 \%\left(1.100 \times 10^{-7}\right)$ \\
Image 3 & $100 \%\left(4.800 \times 10^{-3}\right)$ & $85.7 \%\left(5.415 \times 10^{-7}\right)$ & $47.6 \%\left(4.923 \times 10^{-8}\right)$ & $90.5 \%\left(2.320 \times 10^{-3}\right)$ & $42.9 \%\left(9.555 \times 10^{-7}\right)$ & $80.1 \%\left(1.150 \times 10^{-8}\right)$ \\
Image 4 & $100 \%\left(6.600 \times 10^{-3}\right)$ & $88.9 \%\left(4.368 \times 10^{-7}\right)$ & $88.9 \%\left(5.461 \times 10^{-7}\right)$ & $83.3 \%\left(3.181 \times 10^{-3}\right)$ & $27.8 \%\left(2.741 \times 10^{-7}\right)$ & $83.3 \%\left(1.700 \times 10^{-7}\right)$ \\
Image 5 & $100 \%\left(6.250 \times 10^{-3}\right)$ & $100 \%\left(6.875 \times 10^{-7}\right)$ & $100 \%\left(6.875 \times 10^{-7}\right)$ & $100 \%\left(2.936 \times 10^{-3}\right)$ & $100 \%\left(2.688 \times 10^{-6}\right)$ & $100 \%\left(1.995 \times 10^{-7}\right)$ \\
Image 6 & $100 \%\left(6.300 \times 10^{-3}\right)$ & $97.6 \%\left(6.167 \times 10^{-6}\right)$ & $85.4 \%\left(2.418 \times 10^{-6}\right)$ & $87.8 \%\left(2.603 \times 10^{-3}\right)$ & $80.5 \%\left(1.280 \times 10^{-7}\right)$ & $80.0 \%\left(1.090 \times 10^{-7}\right)$ \\
\hline Mean & $100 \%\left(5.807 \times 10^{-3}\right)$ & $90.5 \%\left(3.920 \times 10^{-6}\right)$ & $72.2 \%\left(2.598 \times 10^{-6}\right)$ & $93.6 \%\left(2.607 \times 10^{-3}\right)$ & $75.2 \%\left(1.147 \times 10^{-6}\right)$ & $85.1 \%\left(1.018 \times 10^{-7}\right)$ \\
Std dev. & $0\left(7.065 \times 10^{-4}\right)$ & $6.87 \%\left(6.146 \times 10^{-6}\right)$ & $19.2 \%\left(4.619 \times 10^{-6}\right)$ & $7.37 \%\left(0.400 \times 10^{-4}\right)$ & $32.2 \%\left(9.843 \times 10^{-7}\right)$ & $6.75 \%\left(7.849 \times 10^{-8}\right)$ \\
\hline
\end{tabular}

TABLE II

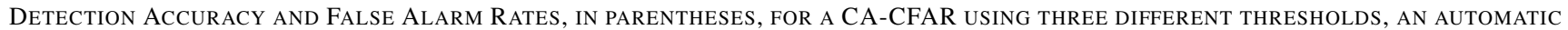
THRESHOLD SELECTION METHOD AND AN ADVANCED OTSU'S BASED METHOD VERSUS THE PROPOSED METHOD.

\begin{tabular}{ccccc}
\hline Image & Automatic threshold & Standard Otsu's & Advanced Otsu's & CA-CFAR SA \\
Image 1 & $88.2 \%\left(8.550 \times 10^{-8}\right)$ & $82.4 \%\left(1.443 \times 10^{-2}\right)$ & $82.4 \%\left(7.695 \times 10^{-7}\right)$ & $82.4 \%\left(1.050 \times 10^{-8}\right)$ \\
Image 2 & $88.4 \%\left(2.581 \times 10^{-7}\right)$ & $86.0 \%\left(3.371 \times 10^{-3}\right)$ & $90.7 \%\left(7.643 \times 10^{-8}\right)$ & $83.7 \%\left(1.100 \times 10^{-7}\right)$ \\
Image 3 & $85.7 \%\left(9.555 \times 10^{-8}\right)$ & $71.4 \%\left(8.310 \times 10^{-3}\right)$ & $76.1 \%\left(3.810 \times 10^{-7}\right)$ & $80.1 \%\left(1.150 \times 10^{-8}\right)$ \\
Image 4 & $88.9 \%\left(3.700 \times 10^{-7}\right)$ & $100 \%\left(1.930 \times 10^{-2}\right)$ & $100 \%\left(9.060 \times 10^{-7}\right)$ & $83.3 \%\left(1.700 \times 10^{-7}\right)$ \\
Image 5 & $100 \%\left(1.005 \times 10^{-7}\right)$ & $100 \%\left(1.510 \times 10^{-2}\right)$ & $100 \%\left(4.409 \times 10^{-7}\right)$ & $100 \%\left(1.995 \times 10^{-7}\right)$ \\
Image 6 & $95.1 \%\left(1.21 \times 10^{-7}\right)$ & $78.0 \%\left(2.582 \times 10^{-3}\right)$ & $78.0 \%\left(2.755 \times 10^{-7}\right)$ & $80.0 \%\left(1.090 \times 10^{-7}\right)$ \\
\hline Mean & $91.1 \%\left(1.718 \times 10^{-7}\right)$ & $86.3 \%\left(1.051 \times 10^{-2}\right)$ & $87.9 \%\left(4.749 \times 10^{-7}\right)$ & $85.1 \%\left(1.018 \times 10^{-7}\right)$ \\
Std dev. & $4.92 \%\left(1.163 \times 10^{-7}\right)$ & $11.7 \%\left(6.814 \times 10^{-3}\right)$ & $10.6 \%\left(3.102 \times 10^{-7}\right)$ & $6.75 \%\left(7.849 \times 10^{-8}\right)$ \\
\hline
\end{tabular}

drop in FAR. When comparing it to the third best method, the advanced Otsu's thresholding, the $2 \%$ drop in DA is compensated by a fourfold increase in FAR performance. The low standard deviation values imply that over a number of test images the proposed method had the most consistent FAR and third most consistent DA results. It should be noted that the proposed method did discard a few correct ships which suggest that future work should identify an adjustment to threshold manifold evaluation and in order to identify when thresholds over correct detections have been increased too much.

\section{CONCLUSION}

To improve a country's MDA a variety of different technologies must be used to monitor ships. Whilst conventional transponder based systems are useful when the ship is cooperative, alternative means of ship detection are required in some case. The usage of SAR imagery for the detection of ships at sea is a well studied topic in literature and the methods range from global thresholding methods to statistical estimation of local windows around ships. A novel extension to a popular ship detection prescreening technique known as CA-CFAR was presented in this paper. The proposed method extends a single scalar threshold to a threshold manifold. To help with the selection of threshold values, the paper presents the SA optimization method in conjunction with a ship distribution map which highlights the most traversed areas in the ocean. This ship distribution map can be generated from any number of ship positional sources and assists in estimating the threshold manifold. The inefficiencies of the SA method are avoided by selecting the results of a low threshold CA-CFAR processed image as input along with bounding the required threshold values. Once an acceptable threshold manifold is generate it is applied to the input SAR image using the extended CA-CFAR method and the results are compared to known ship locations.

The proposed method was compared a number of other methods: conventional CA-CFAR and GO-CFAR prescreening at various thresholds; a standard Otsu's prescreening method; an automatic threshold selection method; and an advanced Otsu's method. The proposed method had a DA of $85.1 \%$ with the lowest reported FAR $1.01 \times 10^{-7}$ of all the methods tested. Furthermore, the results indicate that the method provides consistent performance across a number of test images with the method providing the third lowest standard deviation for DAs and the lowest standard deviation for FARs. The flexibility introduced allows for a multitude of methods to be used in places of the initial prescreening, secondary CFAR prescreening and manifold generation. The results indicate that the method still has room for improvement by altering the sensitivity of the SA evaluation function to identify when manifold threshold values for correct detections have been increased too much.

\section{ACKNOWLEDGMENT}

The authors would like to thank the South African Maritime Safety Authority (SAMSA) for allowing access to the LRIT data used in this paper. 


\section{REFERENCES}

[1] G. Galdorisi and R. Goshorn, "Bridging the Policy and Technology Gap: A Process to Instantiate Maritime Domain Awareness," in Proc. of MTS/IEEE OCEANS 2005, Sep 2005, pp. 1-8.

[2] "Maritime security policy," White Paper, National Security Presidential Directive/Homeland Security Presidential Directive, NSPD-41/HSPD13, 2004.

[3] J. Statsny et al., "A novel adaptive synthetic aperture radar ship detection system," in Proc. of MTS/IEEE OCEANS 2011, Sep 2011, pp. 1-7.

[4] W. Kleynhans, B. P. Salmon, C. P. Schwegmann, and V. Seotlo, "Ship Detection in South African oceans using a combination of SAR and historic LRIT data," in Geoscience and Remote Sensing Symposium, 2013. IGARSS 2013. IEEE International, 2013, pp. 1521-1524.

[5] C. P. Schwegmann, W. Kleynhans, and B. P. Salmon, "Ship Detection in South African oceans using SAR, CFAR and a Haar-like Feature Classifier," in Geoscience and Remote Sensing Symposium, 2014. IGARSS 2014. IEEE International, 2014, pp. 557-560.

[6] C. Schwegmann, W. Kleynhans, and B. P. Salmon, "Simulated Annealing CFAR Threshold Selection for South African Ship Detection in ASAR imagery," in Geoscience and Remote Sensing Symposium, 2014. IGARSS 2014. IEEE International, 2014, pp. 561-564.

[7] D. J. Crisp, "The State-of-the-Art in Ship Detection in Synthetic Aperture Radar Imagery," Australian Department of Defence, Edinburgh, Australia, Tech. Rep. DSTO-RR-0272, 2004.

[8] K. El-Darymli, P. McGuire, D. Power, and C. Moloney, "Target detection in synthetic aperture radar imagery: a state-of-the-art survey," Journal of Applied Remote Sensing, vol. 7, no. 1, pp. 1-35, Mar. 2013.

[9] F. B. D. Stagliano, A. Lupidi, "Ship detection from sar images based on CFAR and wavelet transform," in 2012 Tyrrhenian Workshop on Advances in Radar and Remote Sensing (TyWRRS), Sep. 2012, pp. 5358.

[10] F. Bi, F. Pang, B. Zhu, and L. Chen, "A cascaded false-alarm elimination method for accurate ship detection in SAR images," in Radar Conference 2013, IET International, 2013, pp. 1-4.

[11] W. An, C. Xie, and X. Yuan, "An Improved Iterative Censoring Scheme for CFAR Ship Detection With SAR Imagery," IEEE Transactions on Geoscience and Remote Sensing, vol. 52, no. 8, pp. 4585-4595, Aug. 2014.

[12] L. M. Chen, Digital Functions and Data Reconstruction Digital-Discrete Methods. NY, New Jersey: Springer, 2013.

[13] L. Chen, "Discrete Surfaces and Manifolds: A Potential tool to Image Processing," http://www.tc18.org/subfields/topology/DSM_GW.pdf, accessed: 2015-01-05.

[14] J. B. Tenenbaum, V. de Silva, and J. C. Langford, "A Global Geometric Framework for Nonlinear Dimensionality Reduction," Science, vol. 290, no. 1, pp. 2319-2323, Dec. 2000.

[15] S. Kirkpatrick, C. D. Gelatt, and M. P. Vecchi, "Optimization by Simulated Annealing," International Journal of Applied Earth Observation and Geoinformation, vol. 220, pp. 671-680, 1983.

[16] L. Ingber, "Simulated annealing: Practice versus theory," Mathematical and Computer Modelling, vol. 18, no. 11, pp. 29-57, 1993.

[17] S. Sivanandam and S. Deepa, Introduction to Genetic Algorithms. Heidelberg, Berlin: Springer-Verlag, 2008.

[18] K. Shojaee, H. G. Shakouri, and M. B. Taghadosi, Simulated Annealing, Theory with Applications: Importance of the Initial Conditions and the Time Schedule in the Simulated Annealing, Chapter 12. Croatia: Sciyo, 2010.

[19] M. Schwartz, Encyclopedia of Coastal Science. New York, NJ: Springer, 2005.

[20] P. Lombardo, M. Sciotti, and L. M. Kaplan, "SAR prescreening using both target and shadow information," in 2001 IEEE Proc. Radar Conf., Atlanta, United States of America, May 2001, pp. 147-152.

[21] R. C. Gonzalez and R. E. Woods, Digital Image Processing, 3rd ed. Upper Saddle River, NJ: Prentice Hall, 2008.

[22] M. M. Awad, "A Morphological Model for Extracting Road Networks from High-Resolution Satellite Images," Journal of Engineering, vol. 2013, no. 2013, pp. 1-9, Jan. 2013.

[23] N. Otsu, "A Threshold Selection Method from Gray-Level Histograms," IEEE Transactions on Systems, Man, and Cybernetics, vol. 9, pp. 62-66, Nov. 1979.

[24] M. Messina, M. Greco, L. Fabbrini, and G. Pinelli, "Modified Otsu's algorithm: A new computationally efficient ship detection algorithm for SAR images," in Advances in Radar and Remote Sensing (TyWRRS), 2012 Tyrrhenian Workshop on, Sep 2012, pp. 262-266.

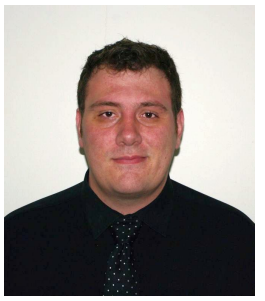

Colin Schwegmann received a B.Eng and an M.Eng (Computer Engineering) from the University of Pretoria, South Africa in 2012 and 2014 respectively. He is currently a Ph.D (Computer Engineering) candidate at the University of Pretoria, South Africa and is on a studentship at the Remote Sensing Research Unit, Council of Scientific and Industrial Research (CSIR) in Pretoria, South Africa. His research areas include SAR and image processing, machine learning, computer vision and Android application development.

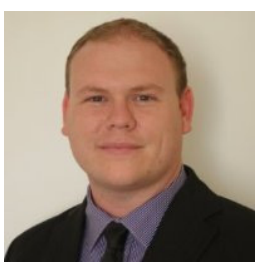

Dr. Waldo Kleynhans received a B.Eng., M.Eng. and $\mathrm{Ph} . \mathrm{D}$. (Electronic Engineering) from the University of Pretoria, South Africa as well as an MBA from Heriot-Watt University, Scotland. He is currently a principal researcher with the Remote Sensing Research Unit at the Council for Scientific and Industrial Research in Pretoria, South Africa and is also affiliated with the University of Pretoria, South Africa and San Diego State University, San Diego, USA. Before joining the CSIR in 2008, he worked in the telecommunications industry as an RF consultant. His research interests include remote sensing, time-series analysis, wireless communications, statistical detection and estimation theory as well as machine learning.

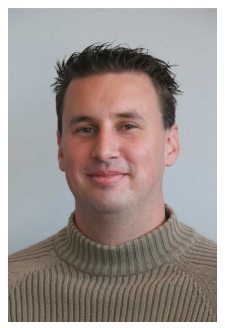

Dr. Brian Salmon received an M.Eng degree in electronic engineering and a Ph.D. degree in electronic engineering from the University of Pretoria, South Africa in 2008 and 2012 respectively. He is presently a postdoctoral research fellow in the School of Engineering at the University of Tasmania. He was the lead author of the paper that won the IEEE Geoscience and Remote Sensing Society 2012 Symposium prize paper award. His research interests are information theory, coding theory, machine learning and graph theory. 